Review

\title{
Disclosing the truth to terminal cancer patients: a discussion of ethical and cultural issues
}

\author{
G.A. Kazdaglis, ${ }^{1}$ C. Arnaoutoglou, ${ }^{2}$ D. Karypidis, ${ }^{3}$ G. Memekidou, ${ }^{4}$ G. Spanos ${ }^{5}$ and O. Papadopoulos ${ }^{6}$
}

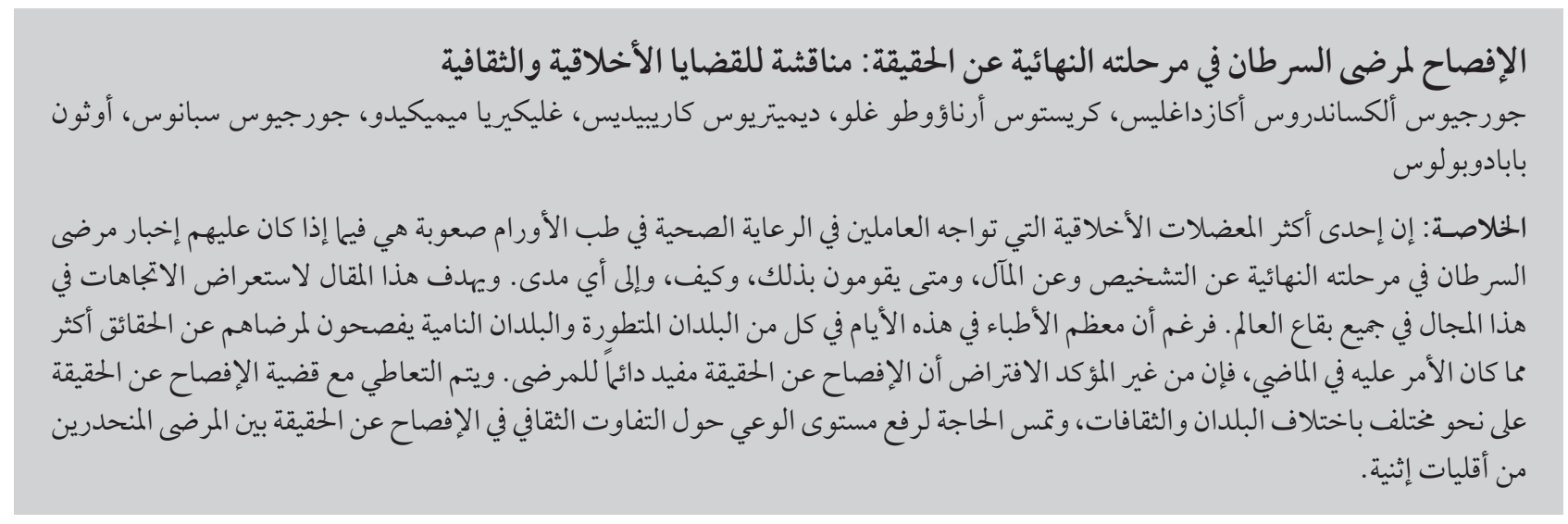

ABSTRACT One of the most difficult ethical dilemmas facing health care professionals working in oncology is whether, when, how and how much to tell terminal cancer patients about their diagnosis and prognosis. The aim of this article is to review the trends in this issue worldwide. While a majority of physicians in both developed and developing countries tell the truth more often today than in the past, the assumption that truth-telling is always beneficial to patients can be questioned. The issue of truth-telling is still approached differently in different countries and cultures and there is a need for an increased awareness of cultural differences to truth-telling among patients from ethnic minorities.

Divulgation de la vérité aux patients atteints d'un cancer en phase terminale : discussion sur les problèmes éthiques et culturels

RÉSUMÉ Un des dilemmes éthiques les plus difficiles auxquels sont confrontés les professionnels de la santé qui travaillent dans le domaine de la cancérologie est de définir s'il faut annoncer le diagnostic et le pronostic aux patients atteints d'un cancer en phase terminale, et à quel moment, de quelle manière et dans quelle mesure leur en parler. L'objectif de cet article est d'étudier la tendance dans ce domaine au niveau international. Même si la majorité des médecins, aussi bien dans les pays développés que dans les pays en développement, disent plus souvent la vérité aujourd'hui que par le passé, l'idée selon laquelle dire la vérité est toujours bénéfique pour le patient donne matière à réflexion. Le fait de dire la vérité ou non est abordé de manière différente en fonction des pays et des cultures, et une plus grande conscience des différences culturelles est nécessaire avant de dire la vérité aux patients issus de minorités ethniques.

'Department of General Surgery, Saint Savvas Oncology Hospital, Athens, Greece (Correspondence to G.A. Kazdaglis: kazdaglis@inbox.com). ${ }^{2}$ Division of Gynaecologic Oncology, Department of Obstetrics and Gynaecology, Darlington Memorial Hospital, Darlington, United Kingdom. ${ }^{3}$ Eastern Virginia Medical School, Norfolk, Virginia, United States of America.

${ }^{4}$ Department of Oncology, Athens Naval and Veteran Hospital, Athens, Greece.

${ }^{5}$ Eurodiagnosis Diagnostic Centre, Thessaloniki, Greece.

${ }^{6}$ Department of Plastic Surgery, A. Sygros Hospital of Dermatologic Diseases, University of Athens, Athens, Greece.

Received: 21/02/08; accepted: 03/04/08 


\section{Introduction}

Cancer is a complicated illness, which for many years and in many cultures was perceived as incurable. Cancer meant death, and there was a tendency to hide the diagnosis from the patient, who was subconsciously excluded from life [1]. This situation has changed: currently health professionals generally prefer to inform cancer patients about their illness, albeit more often in some countries than others [2]. However, deception is still sometimes used by members of the health care team while treating cancer patients [3]. It might be intentional or unintentional and, apart from obvious lies, it can involve half-truths and controlled release of information.

There is no doubt that the diagnosis of a life-threatening disease such as cancer is devastating for patients, and informing such a patient of the diagnosis has been described as "dropping a bomb" [4]. Health care professionals are the ones who have to carry out the task of providing information to patients about their diagnosis and prognosis. This task becomes more complicated if the patient receiving the cancer diagnosis belongs to an ethnic minority which may have different cultural beliefs about personal autonomy and death. The importance of ethnic background in attitudes and practices in health is widely acknowledged. Ethnic minorities are present in most countries and therefore cultural issues such as the one addressed in the present article, should be assessed and taken into consideration.

One of the most difficult ethical dilemmas that health care professionals working in oncology settings face is whether, when, how and how much to tell cancer patients about their diagnosis and prognosis. The issue of truth-telling is still approached differently depending on the country and culture. The majority of physicians, in both developed and developing countries, tell the truth more often today than in the past.
More attention ought to be drawn to the evidence whether truth-telling has indeed a positive impact on patients' quality oflife or is just assumed by health care professionals to be beneficial to patients. Furthermore, an increased awareness of cultural differences regarding truth-telling is needed to achieve optimal health outcomes among different communities.

This article aimed to discuss the issues involved in the disclosure of sensitive information concerning the health and prognosis of terminally-ill patients in an effort to enhance the understanding of health care professionals.

\section{Sources}

The general aim of the study was to identify the trends in the issue by searching the contemporary literature databases (PubMed, WHOLIS, Cochrane Library) for articles published in English from 1960 to 2005 using the terms "palliative care", "cancer", "neoplasm", "oncology", "tumour", "truth telling", "culture", "bad breaking news", "deception", "ethnic origin", "ethics" and "quality of life" as keywords.

The resulting list of 1507 articles was screened to eliminate duplicates and references that were not specifically relevant to our topic. Studies that involved diseases other than cancer, but which also included a substantial proportion of cancer cases, were included in this theoretical discussion. Evaluation of the abstracts resulted in 234 references that matched the topic. All 234 were fully assessed and analysed by at least 2 authors.

\section{The issue of truth- telling}

Since ancient times, philosophers and religious thinkers have debated issues concerning deception and truth- telling. They have generally regarded truth as preferable, if not indispensable, to human relationships, and deception as something that, at the very least, needs justification [5].

Truth-telling is a complicated business. From a health care perspective, it may be defined as total openness about the diagnosis and prognosis. For health care professionals the difficulty and the dilemma of whether to tell the truth or not, becomes obvious when having to break bad news, especially if it involves cancer. Buckman describes bad news as any information likely to alter drastically and negatively the patient's view of his or her own future [6].

A successful relationship between patients and health care providers depends on the establishment of trust, which is strongly connected with truthful communication [7]. On that basis, truth-telling is considered to be an ethical issue as well as a moral obligation by a large number of health care professionals [2]. Similarly, patients expect that their health care providers will tell them the truth, just as health care providers expect that their patients will tell them the truth. It has also been suggested that disclosure of truth fosters trust and is an essential element of the respect owed to the patient as a person [8]. Therefore, the disclosure of truth aids the whole process of establishing an optimum patient-carer relationship.

Another purpose of information giving is to reduce uncertainty and to provide a basis for action [9]. It enables patients to make informed choices about their own health care and plan for their future. It prevents harm, as patients who are not informed about their situation may fail to get the medical support that they need [8]. Better knowledge affects patient and family responses in different clinical situations [9], engaging patients in their care and securing their collaboration, leading to patients' adherence to the therapeutic or palliative schemes $[10,11]$. Uncertainty, 
doubt or misinterpretation do not offer a foundation for proper psychological support [12].

On the other hand, when disclosing the truth, there is always the risk of shattering a patient's hopes and dreams. Hope seems to an essential mechanism for coping with cancer [13] and it can be jeopardized by excessive knowledge and detail. Revealing the stark reality of a cancer diagnosis is often perceived as the passing of a death sentence by the members of the health care team and this may also be the case for patients and their relatives [14]. One the most common concerns of health care professionals is whether they are justified if harm results from telling the truth.

Certainly, patients seem to be heterogeneous in their information requirements. Full, uncensored disclosure, which is insisted upon by one patient, may be utterly undesirable to another [2]. It can be argued that it is important to take into account the individual needs of every patient, recognizing that full information disclosure may not necessarily be desired or helpful for some patients at a specific time, even though the situation could change later.

It is important to consider the ethical issue of truth-telling from both perspectives. There is no clear distinction between right and wrong and therefore a tenuous balance exists.

\section{Three perspectives} on truth-telling

\section{The physicians' perspective}

In the field of oncology, physicians are unavoidably forced to break bad news [15]. It is likely that, even in countries where full disclosure is the norm, lies, perhaps "white" (beneficial) lies, are told from time to time [3].

Despite the abundance of articles, research and training about how to perform the difficult task of giving a cancer diagnosis and presenting treatment options [16], many physicians feel uncomfortable and unprepared for the interaction. There is a general consensus that breaking bad news, while an important part of a physician's job, is not an easy task [4]. Therefore, physicians have a duty to improve their communication skills in order to assist the patient as much as possible at this difficult time.

\section{The nurses' perspective}

There are few studies about nurses and truth-telling, because communicating about new medical diagnoses and their prognosis is traditionally a physician's responsibility [17]. According to Faulkner, the person delivering the bad news should be someone whom the patient trusts and can feel most comfortable with [18]. Even though for many patients this person is their nurse, breaking bad news is not a common practice for nurses, with the exception of nurses in the United Kingdom (UK) [14].

Many nurses believe that their role in communicating with patients is secondary to the physician's role [1]. This is probably an easy way of disclaiming responsibility for a task that clearly no health professional wishes to do. Nurses often express feelings of powerlessness and they experience an ethical conflict when they are aware of non-disclosure of information by physicians [19].

Nurses are constantly communicating with patients [20]. Especially in cancer nursing, communication is considered one of the most important aspects. "Picking up the pieces" is a common scenario for the great majority of nurses involved in cancer care [21]. Since their role is to advocate for the patient in all situations, they too have a duty to be more prepared and to contribute to ensuring that patients' true wishes are respected, or at least heard. The provision of health services is not a "one-man show" and all health professionals have responsibilities.

\section{The patients' perspective}

Two main issues are presented for patients: first, the response to the stressful event of learning the truth and, second, adaptation to the knowledge of a future with a chronic or even terminal disease.

Patients seem to remember situations where bad news is given in detail, even some considerable time after the event [18]. A cancer diagnosis changes a person's life and reduces his or her opportunities drastically [22]. The media constantly report complaints about the way patients have received bad news. Disclosing the truth by just giving clinical facts, without concern for the sensitivity with which it is done or the obligation to support the patient and assist him/her in decision-making can result in patients being as upset as if they were lied to [4].

But what do patients really want to know? The literature suggests that many want to know the truth about their diagnosis [8]. However, there is evidence from the UK that a substantial minority of patients (13\%) prefers to "leave it up to the doctor" or "to have information only if it is good" [23]. In countries where truth disclosure is not a common practice the proportion of patients having this view is even higher, with one example of almost half the terminally-ill patients in a study in Spain [24].

What is important to note is that, in most studies showing that patients prefer to be informed, the patients interviewed already knew about their illness and therefore their opinions are likely to be biased. Consequently, there is a possibility that fewer patients want to know in detail about their illness than is believed by health professionals.

\section{Changing attitudes to truth-telling}

During the past few decades, health professionals' attitudes towards the balance between telling and not telling the 
truth has changed dramatically. In 1961 $90 \%$ of a sample of 219 physicians in the United States of America (USA) reported that they preferred not to disclose a diagnosis of cancer to their patients [25]. Almost 20 years later, $97 \%$ of 264 physicians were open about telling cancer patients their diagnosis [26]. This indicates a complete reversal of attitude in the USA.

It used to be considered inhumane and damaging to the patient to disclose a diagnosis of cancer when the treatment prospects were bleak. Advances in treatment have since altered the course of many cases of cancer, so that it is much easier now to offer patients hope at the time of diagnosis and this has also removed the "wall of silence" that surrounded the cancer patient [4].

Currently the debate within the USA is not whether patients should be told or not, but how and when to tell them the truth. However, revealing the diagnosis to a patient with terminal cancer is not currently fully accepted in some countries without an AngloSaxon cultural background, such as Japan, China, Greece, Turkey, Spain and Italy. There is a tendency to disclose the truth more often than in the past in these countries, but full openness is still not a common practice $[27,28]$. However, truth-telling is considered an ethical issue as well as a moral obligation by health care professionals [2].

\section{International comparisons}

There are many differences among countries regarding health information disclosure practices. The purpose of this article is not to make a detailed comparison of rates of disclosure nor to judge whether one practice is better than another.

In the USA, as already mentioned, the majority of patients are told of their diagnosis. The same is true in England
[29], Canada [8] and Finland [30]. It appears that in these countries health care professionals have little choice about whether or not to tell patients, as current disclosure policies recommend that they should provide full information. Furthermore, the patients' right to participate in decisions about their care is safeguarded by legislation. However, is it possible that these countries have moved too far the other way? Have patients now lost the right not to have "the whole truth and nothing but the truth"?

In Japan, family members play a major role in the decision whether a physician should inform a patient with cancer about the true nature of his/her illness. Physicians discuss the cancer diagnosis with the family before discussing it with the patient and commonly comply with the family members' requests. There is a family hierarchy and Japanese patients generally do not become the master of their own cancer treatment. Instead, they deal with their disease while their family takes decisions [31]. Consequently, in Japanese society only a small percentage of physicians (13\%) inform cancer patients about their diagnosis [32].

Similar paternalistic practices are observed in Arab and Islamic cultures. In a survey in Turkey a significant proportion of cancer patients (44\%) did not know their diagnosis [33]. In Lebanon, where legislation allows non-disclosure, nearly half of physicians would usually tell the patient about cancer [34].The great majority of physicians (79\%) in Kuwait would withhold the truth if the patient's family requested them to do so [35], and in Saudi Arabia 75\% of physicians preferred to discuss information with close relatives rather than patients themselves, even when the latter were mentally competent [36].

Withholding the truth from patients appears common in Greece too [37]. The majority of Greek health care professionals believe that relatives should be informed, but when it comes to informing the patients, they are not so sure [1]. Family bonds are strong in Greek society and, in an effort to protect them from despair and feelings of hopelessness, families often exclude patients from the process of information exchange [27].

In a study in Spain $68 \%$ of patients were not informed about their terminal cancer, but the approach is more paternalistic. Only a few physicians take into account the family wishes. Their decision whether to give full information to their patients depends basically on their own views about the ability of the individual patient to cope with the diagnosis [24].

The evidence suggests that the issue of truth-telling in health care-what a terminally ill person wants to hear and how it is told-are is so embedded in the culture that it cannot be seen as simply an ethical dilemma [38]. Although it might be difficult to envisage a situation when not telling the truth will improve patient care, frank disclosure may be considered inappropriate and insensitive, so that in some cultures "bad news" should be told to a family member of the patient who will divulge the information to the patient at an appropriate time and place and in a culturally approved and recognized manner.

\section{Possible justifications for non-disclosure}

Not disclosing the truth to patients about their diagnosis and prognosis can to some extent be justified in underdeveloped countries. In countries with more-advanced health systems, patients with terminal cancer can be reassured that everything possible will be done for them and that they will die eventually with dignity and without pain. In less-developed countries, where the quality of health care provided is often poor and lacking in palliative care facilities, patients with terminal cancer 
face the prospect of dying in discomfort and pain.

Another criticism of full disclosure hinges on the degree of personal responsibility that health professionals nowadays delegate to patients. In the most developed countries health care professionals are expected to involve patients fully in the decision-making process. However, this can result in the patient being forced to make decisions. Instead of offering guidance, health care professionals nowadays present the available options to the patients and expect them to choose. They may even avoid stating their professional opinion because they are afraid of being blamed by the patient if something goes wrong.

\section{Effects of truth disclosure on cancer patients' quality of life}

Quality of life is a descriptive term and has a broad meaning. In health care settings, it refers to patients' emotional, social and physical well-being and their ability to function in the ordinary tasks of living [39]. Especially in terminal cancer care, where patients have limited remaining life, one of the most important targets is to maintain, and if possible improve, their quality of life. Therefore, when assessing truth disclosure and before coming to any conclusions, health professionals should take into consideration the effect, if any, that it may have on the patients' quality of life.

There does not appear to be much research on the relationship between truth disclosure and quality of life. According to Montazeri at al., in most studies patients' quality of life was assessed after the diagnosis of cancer and after or during each course of treatment, raising questions about the validity of these studies [40]. Two comparative studies have looked at patients' quality of life before and after disclosure of the cancer diagnosis; a study in the UK suggested that "the knowledge of cancer diagnosis does not affect the way in which patients respond to quality of life questionnaires" [40], while a study in Turkey stated that "honest disclosure of truth does not worsen any dimension of quality of life in general or emotional functioning in particular" [33]. A systematic review trying to answer the question whether telling the truth has a positive or negative effect on the patients' psychological distress came to no conclusions, as no studies fully met the inclusion criteria [41]. The authors stated that the available evidence was contradictory and more rigorous studies were needed to provide the evidence.

In the absence of evidence as to whether disclosing the truth or not affects patients' quality of life, it is important to reconsider the argument that telling the truth is universally "good" for the patient. Although truth-telling is preferable from a philosophical point of view, when it comes to real life, theories cannot be applied so easily and may not have the results expected.

\section{Conclusions}

The duty of all health professionals is first and foremost to the patient. Being truthful involves nurses and doctors finding out what patients' information needs are. Further research on the subject is needed in order to identify how patients' interests are best served. Until we know more, a cautionary note is indicated.

Although desirable, full disclosure and openness in every case may be unrealistic. A consensus that lies somewhere between the 2 extremes may be more appropriate. In Asia and south-east Europe, where revealing the diagnosis is difficult because of cultural issues, health professionals should be more willing to answer patients' questions truthfully when they indicate a preference to know, even if they do not express it verbally. In the USA and the UK, in contrast, health professionals should perhaps adopt a more cautious attitude. Respect for the patient should include identification of those who wish to know less and complying with their choice. Finally, all health care professionals, in whatever country, should bear in mind that ethnic minorities with different cultural backgrounds and different attitudes to disclosure are present in almost every society around the world.

Rather than trying to guess patients' preferences, a possibility would be to ask them well before any diagnosis is made. Just as patients are asked for their preferences about resuscitation should it became necessary or asked to give consent before surgery, they could similarly be asked to indicate their preferences about information disclosure. A question of this type should be addressed as a matter of course to all patients, not only the ones with possible cancer. Although this idea is not currently easy to put in practice, it is an alternative that deserves further consideration.

This article has explored the dilemma of how to always satisfy the 2 ethical principles of veracity (truthfulness) and beneficence (doing good). There is no answer that "fits" all patients in all countries, but it is valuable for health professionals to have a broader understanding of the issues and to treat each patient as an individual.

\section{Acknowledgement}

The authors would like to thank $\mathrm{Mr}$ Iraklis Zografos, historian and professor of English, for his support and contribution towards writing the above manuscript. 


\section{References}

1. Georgaki S et al. Nurses' attitudes towards truthful communication with patients with cancer. Cancer nursing, 2002, 25(6):436-41.

2. Nekolaichuk CL, Bruera E. On the nature of hope in palliative care. Journal of palliative care, 1998, 14(1):36-42.

3. Tuckett A. "Bending the truth": professionals' narratives about lying and deception in nursing practice. International journal of nursing studies, 1998, 35(5):292-302.

4. Baile WF et al. SPIKES-a six-step protocol for delivering bad news: application to the patient with cancer. Oncologist, 2000, 5(4):302-11.

5. Littlejohn JE. Lying, deceit, fraud: do they ever belong in your nursing practice? Maryland nurse, 1999, 18(3):5-6.

6. Buckman R. How to break bad news. Basingstoke, UK, Papermac, 1992.

7. Bishara $\mathrm{E}$ et al. Is there a relationship between psychological well-being and patient-carers consensus? A clinical pilot study. Journal of palliative care, 1997, 13(4):14-22.

8. Hebert PC et al. Bioethics for clinicians: 7.Truth telling. Canadian Medical Association journal, 1997, 156(2):225-8.

9. Erlen JA. Should the nurse participate in planned deception? Orthopaedic nursing, 1995, 14(2):62-6.

10. Ruhnke GW et al. Ethical decision making and patient autonomy: a comparison of physicians and patients in Japan and the United States. Chest, 2000, 118(4):1172-82.

11. Eraker S, Kirscht J, Becker M. Understanding and improving patient compliance. Annals of internal medicine, 1984, 100:258-68.

12. Freedman TG. Prescriptions for health providers: from cancer patients. Cancer nursing, 2003, 26(4):323-30.

13. Schattner A. What do patients really want to know? Quarterly journal of medicine, 2002, 95(3):135-6.

14. Costello J. Is it always ethical for health professionals to tell the truth about cancer? Cancer nursing practice, 2004, 3(8):25-30.

15. Ptacek JT, Eberhardt TL. Breaking bad news: a review of the literature. Journal of the American Medical Association, 1996, 276(6):496-502

16. Garg A, Buckman R, Kason Y. Teaching medical students how to break bad news. Canadian Medical Association journal, 1997, 156(8):1159-64.

17. Clark AP, Volker DL. Legal and ethical dimensions of CNS practice: truthfulness. Clinical nurse specialist, 2003, 17(1):17-8.

18. Faulkner A. When the news is bad: a guide for health professionals. Cheltenham, UK, Nelson Thornes, 1998.

19. Blake C, Guare RE. Nurses' reflections on ethical decision making: implications for leaders. Journal of the New York State Nurses' Association, 1997, 28(4):13-6.

20. Wallace PR. Improving palliative care through effective communication. International journal of palliative nursing, 2001, 7(2):86-90

21. Higgins D. Breaking bad news in cancer care. Part 2: practical skills. Professional nurse, 2002, 17(11):670-71.

22. Kirk P, Kirk I, Kristjanson JL. What do patients receiving palliative care for cancer and their families want to be told? A Canadian and Australian qualitative study. British medical journal, 2004, 328:1343-7.

23. Jenkins V, Fallowfield L, Saul J. Information needs of patients with cancer: from a large study in UK cancer centres. British journal of cancer, 2001, 84(1):48-51.
24. Centeno-Cortes C, Nunez-Olarte JM. Questioning diagnosis disclosure in terminal cancer patients: a prospective study evaluating patients' responses. Palliative medicine, 1994, 8(1):39-44.

25. Oken D. What to tell cancer patients: a study of medical attitudes. Journal of the American Medical Association, 1961, 175:1120-8

26. Novack DH et al. Changes in physician's attitude toward telling the cancer patient. Journal of the American Medical Association, 1979, 241(9):897-900.

27. Mystakidou $\mathrm{K}$ et al. Cancer information disclosure in different cultural contexts. Supportive care in cancer, 2004, 12(3):147-54.

28. Elwyn TS et al. Cancer disclosure in Japan: historical comparisons, current practices. Social science \& medicine, 1998 46(9):1151-63

29. Seale C. Communication and awareness about death: a study of a random sample of dying people. Social science and medicine, 1991, 32(8):943-52.

30. Sainio C, Lauris S, Eriksson E. Cancer patients' views and experiences of participation in care and decision making. Nursing ethics, 2001, 8(2):97-113.

31. Mizuno M, Onishi C, Ouishi F. Truth disclosure of cancer diagnosis and its influence on bereaved Japanese families. Cancer nursing, 2002, 25(5):396-403.

32. Tanida N. Japanese attitudes towards truth disclosure in c ancer. Scandinavian journal of social medicine, 1994, 22(1):50-7.

33. Bozcuk $\mathrm{H}$ et al. Does awareness of diagnosis make any difference to quality of life? Determinants of emotional functioning in a group of cancer patients in Turkey. Supportive care in cancer, 2002, 10(1):51-7.

34. Hamadeh GN, Adib SM. Cancer truth disclosure by Lebanese doctors. Social science \& medicine, 1998, 47(9):1289-94.

35. Qasem AA et al. Disclosure of cancer diagnosis and prognosis by physicians in Kuwait. International journal of clinical practice, 2002, 56(3):215-8.

36. Mobeireek AF et al. Communication with the seriously ill: physicians' attitudes in Saudi Arabia. Journal of medical ethics, 1996, 22(5):282-5.

37. Mystakidou K et al. Disclosure of diagnostic information to cancer patients in Greece. Palliative medicine, 1996, 10(3):195200.

38. Beyene Y. Medical disclosure and refugees. Telling bad news to Ethiopian patients. Western journal of medicine, 1992; 157:328-32.

39. Soni MK, Cella D. Quality of life and symptom measures in oncology: an overview. American journal of managed care, 2002, 8(18):560-73.

40. Montazeri A et al. Does knowledge of cancer diagnosis affect quality of life? A methodological challenge. BMC cancer, 2004, $4(1): 21-6$.

41. Leliopoulo C, Wilkinson SM, Fellowes D. Does truth-telling improve psychological distress of palliative care patients? A systematic review. York, England, University of York NHS Centre for Review and Dissemination, 2002 (http://www.crd.york. ac.uk/CRDWeb/ShowRecord.asp?ID=12002008114, accessed 6 November 2009). 